\title{
Quali-EDU: Um processo de avaliação da qualidade de software educacional
}

\author{
Jefferson Felipe Silva de Lima ${ }^{1}$, Vivianne de Queiroz Leal ${ }^{2}$, Reinaldo Cézar de \\ Morais Gomes ${ }^{3}$, Luciana de Queiroz Leal Gomes ${ }^{1}$ \\ ${ }^{1}$ Universidade Estadual da Paraíba, Campina Grande, PB - Brasil \\ ${ }^{2}$ Ministério Público da Paraíba - João Pessoa, PB - Brasil \\ ${ }^{3}$ Universidade Federal de Campina Grande - Campina Grande, PB - Brasil \\ mru2182@gmail.com,vivianne@mp.pb.gov.br, reinaldo@dsc.ufcg.edu.br, \\ lucianaecct.uepb.edu.br
}

\begin{abstract}
The use of tools, as educational software and educational games, has shown a significant improvement in the understanding and fixation of the knowledge learned in the classroom. However, there are few initiatives found for the development of such systems in Brazil. We also observed that papers did not considered the quality assessment. Besides, in the situations encountered, the Educational Software end-user presence (the teacher and the student) was discarded. This paper presents Quali-EDU, a quality assessment process for Educational Software product, which brings as main contributions the inclusion of educational aspects on its conception and a greater involvement of the student and the teacher in the evaluation of this software.
\end{abstract}

Resumo. A utilização de ferramentas como softwares educacionais e jogos educacionais, tem demonstrado uma melhoria significativa na compreensão e fixação dos conhecimentos trabalhados em sala de aula, mas ainda são poucas as iniciativas encontradas para o desenvolvimento desse tipo de sistemas no Brasil. Observou-se que a avaliação da qualidade não era considerada na maioria dos trabalhos pesquisados que tratavam do desenvolvimento de Software Educacional. Além disso, poucas iniciativas sugerem a presença do usuário final do Software Educacional (o professor e o aluno) na avaliação. Assim, este trabalho apresenta o Quali-EDU, um processo de avaliação de qualidade do produto de Software Educacional, que traz como contribuições a inclusão de aspectos educacionais na sua concepção e um maior envolvimento do aluno e do professor na avaliação desse software.

\section{Introdução}

O constante avanço das tecnologias disponibilizadas no mercado faz com que aumente, a cada dia, o público que as utiliza. Tecnologias computacionais aliadas ao constante desenvolvimento das tecnologias da informação têm transformado a forma com que a educação é conduzida atualmente [Lou, Abrami e D'apollonia 2001]. É confirmado que, embora as tecnologias computacionais possuam o potencial poderoso e flexível [Scardamalia e Bereiter 1996], experiências anteriores de tecnologias de vanguarda utilizadas nas escolas (rádio, televisão, entre outros), demonstram que a simples 
instalação de hardware não necessariamente irá levar-nos aos resultados desejados em termos de aprendizado.

Considerando a realidade brasileira, para a qual é de grande relevância que o sistema educacional tenha na utilização de softwares educacionais um aparato que venha a facilitar o processo de ensino-aprendizagem, é necessário que seus usuários estejam tecnologicamente preparados para serem: a) Usuários capacitados ante as tecnologias da informação, b) Buscadores, analisadores e avaliadores de Informação, c) Solucionadores de problemas e tomadores de decisões, d) Usuários criativos e eficientes de ferramentas de produtividade, e) Comunicadores, colaboradores, publicadores e produtores [Coutinho e Lisbôa 2011]. Além disto, que o software a ser utilizado atenda às necessidades implícitas e explícitas dos seus usuários e que contribua para esta preparação dos mesmos.

Com relação aos grupos de stakeholders de softwares educacionais, de acordo com Nori, Reddy e Chimalakonda (2014), tem-se os seguintes: (1) alunos, (2) professores, empregadores, sociedade; (3) administradores e (4) gerentes. Ainda, a importância do papel do aluno é reforçada, pois o mesmo pode ser visto como um produto do Sistema Educacional, bem como um consumidor, o beneficiário imediato [Nori, Reddy e Chimalakonda 2014]. Acrescentem-se a estas afirmações o fato de que muitas vezes professores e alunos não participam da avaliação do software educacional. Por não considerar os interesses e as necessidades de todos os participantes em sala de aula no projeto de atividades pedagógicas, tensão é gerada entre as crenças dos professores, investigadores em educação, projetistas de software e práticas de ensino ao instruir uma classe [Nussbaum e Infante 2013].

De acordo com o apresentado até aqui, o presente trabalho propõe o Quali-EDU, um Processo de Avaliação da Qualidade do Produto de Software Educacional, baseado nas Normas da ISO 9126 e ISO 14558, as quais tratam da definição de atributos necessários para avaliação e a avaliação do produto de software baseados nestes, respectivamente. Ainda, propõe-se a avaliar se o software se enquadra no contexto a ser aplicado, contanto com professores e alunos como avaliadores. Para o desenvolvime nto deste trabalho, inicialmente foi realizada uma pesquisa de caráter bibliográfico. Em seguida, foi realizado um estudo de caso onde o Quali-EDU foi usado na avaliação de um software educacional, o Tux Math, mediante a aplicação de questionário disponibilizado pelo modelo, o que caracteriza uma pesquisa qualitativa.

Isto posto, objetiva-se avaliar, por meio da utilização prática do Quali-EDU em instituições de ensino da cidade de Sumé-Paraîba, a viabilidade da inclusão de um processo para a avaliação de softwares educacionais nesse contexto. Nesse sentido o presente artigo se desenvolve, estruturando-se, além desta Introdução, nas seguintes seções: 2. Avaliação de Qualidade de Software Educacional e Normas ISO na Avaliação do Produto de Software, que apresenta uma revisão da literatura relacionada; 3. QualiEDU e a Avaliação de Software Educacional, que trata da apresentação do processo proposto; 4. Avaliação utilizando o Quali-EDU; e Considerações Finais e Trabalhos Futuros.

\section{Avaliação de Qualidade de Software Educacional e as Normas ISO}

Software educacional é definido como sendo um sistema computacional e interativo, intencionalmente concebido para facilitar a aprendizagem de conceitos específicos [Kelly 
2008]. Segundo Fang (2008), software educacional é um tipo de software designado a facilitar o ensino e a aprendizagem, e possui três propriedades: é um software, uma ferramenta educacional e um mediador entre pensamento e conhecimento. Assim sendo, qualquer software que agregue essas características pode trazer resultados positivos quando utilizado em conjunto com os recursos didáticos já disponíveis em sala de aula.

Um dos problemas capazes de comprometer o desenvolvimento deste tipo de software, pode estar no fato de o âmbito educacional ser diferente dos demais (empresarial, comercial, entre outros), no que se refere à variedade dos atores envolvidos. As visões, os conceitos agregados e as expectativas dos stakeholders do software educacional são distintos, apesar de objetivarem o mesmo fim, que é a educação de qualidade. Isto explica muitas vezes, a baixa popularidade dos softwares educacionais.

Com relação à avaliação da qualidade do software educacional, algumas iniciativas podem ser citadas, a exemplo do ESHTRI Model [Fang 2008] (Educational Software Quality Hierarchy Triangle, em tradução livre Triangulo Hierárquico de Qualidade de Software Educacional) que é um modelo que adequou a norma ISO 9126 para a realidade educacional. O modelo considera 15 (quinze) fatores como significantes e diretamente influenciáveis para a qualidade final do software educacional (Corretude, Confiança, Integridade, Usabilidade, Eficiência na Execução, Manutenção, Testabilidade, Interoperabilidade, Flexibilidade, Reusabilidade, Portabilidade, Instabilidade, Autodescrição, Eficiência, Economia). Para cada fator, métricas diretas (pois facilitam no entendimento e comparação) são associadas, para servirem como base para representações quantitativas.

Rocha e Campos (1993) desenvolveram um método para avaliação da qualidade de software educacional que envolve os conceitos: objetivos de qualidade, fatores de qualidade de acordo com os envolvidos (usuário final, alunos e professores), critérios (atributos primitivos a serem avaliados), processos de avaliação, medidas (grau de presença de um critério em um produto) e medidas agregadas. No entanto, o modelo criado não apresenta um processo para avaliação do software educacional, ou seja, não há um guia de como usar o método nem menção de alguma aplicação realizada.

Finalmente, o Modelo de Design Multimídia de Reeves é um modelo destinado à modelagem de design instrucional de sistemas [Litchfield 1994]. É um típico modelo de design instrucional de sistemas, destinado à educação e treinamento. $\mathrm{O}$ mesmo inclui basicamente 4 (quatro) funções prioritárias: Análise, Design, Produção e Avaliação. Cada fase destas incita a um conjunto de atividades específicas desenvolvidas por um grupo de pessoas na equipe de desenvolvimento. Este modelo, apesar de ser voltado à educação, não consegue contemplar diretamente os usuários envolvidos.

Dos três modelos apresentados, apenas um associa a qualidade aos usuários finais do software educacional. Este, além de outros encontrados nas pesquisas realizadas, a citar, [Nori, Reddy e Chimalakonda 2014] e [Nussbaum e Infante 2013], tratam da inclusão do usuário no processo de produção de softwares educacionais, no entanto não propõem modelo de avaliação da qualidade de software.

\subsection{Normas ISO/IEC 9126 e ISO/IEC 14598}

A família de normas ISO/IEC 9126 trata da qualidade do produto de software, através da análise dos principais itens que possam confirmar a acurácia do software quando este for 
aplicado. Já a família de normas ISO/IEC 14598, aborda a avaliação propriamente dita, de acordo com os critérios estabelecidos na primeira família de normas citada.

A norma ISO 9126 (2002), composta por 4 (quatro) documentos, propõe no primeiro documento, ISO 9126-1, um modelo de qualidade para o produto, descrevendo um conjunto de características de qualidade. Já os outros três documentos (ISO 9126-2, ISO 9126-3 e ISO 9126-4), propõem respectivamente métricas externas (produto em execução), métricas internas (características de projeto e código) e métricas de qualidade em uso (características inerentes ao usuário).

Já a norma ISO 14598 (1998) é dividida em 6 (seis) documentos que estabelecem processualmente as ações que propiciam a análise dos requisitos, especificações, projeto e planejamento, execução e documentação dos resultados. O primeiro documento desta norma fornece uma visão geral do processo, definindo uma estrutura de funcionamento. Já o segundo, trata do planejamento e da gestão, em seguida, no terceiro documento, é abordada a definição do processo para os desenvolvedores. O quarto documento explicita o processo para os adquirentes. Os dois últimos documentos tratam da definição do processo para os avaliadores e a documentação dos módulos respectivamente.

As duas normas apresentadas nesta seção são complementares e podem ser utilizadas em conjunto para a avaliação de softwares. Considerando isto, elas foram escolhidas para a elaboração do Quali-EDU, sendo a ISO 9126 utilizada para especificar as características do modelo de qualidade, agregando a estas algumas características relacionadas aos aspectos educacionais do software e a ISO 14598 utilizada para a definição do processo de avaliação do software educacional.

\section{Quali-EDU e a Avaliação de Software Educacional}

O Quali-EDU foi elaborado com base na família de normas ISO/IEC 9126 e na ISO/IEC 14598, tendo como premissa ser um modelo de qualidade de avaliação de software educacional que esteja o mais próximo possível do usuário final (professor e aluno). As características consideradas nos softwares cuja avaliação da qualidade se realize através do processo Quali-EDU são: Funcionalidade, Confiabilidade, Usabilidade, Eficiência, Manutenibilidade, Portabilidade, Eficácia, Produtividade e Satisfação. Vale a pena ressaltar que todas essas características estão em conformidade com a família ISO/IEC 9126.

Entretanto, ao mesmo tempo em que um software educacional pode auxiliar, também pode prejudicar o processo de ensino-aprendizagem [Giraffa 2000]. Por isso, além das características descritas acima, a característica "Aspectos Educacionais" foi incluída no Quali-EDU, composta pelas seguintes sub-características: Coerência, Contextualização, Motivação, Satisfatoriedade e Adequação à faixa etária.

Para cada característica, existe um template específico no Quali-EDU, que contempla as sub-características e também um questionário que visa auxiliar a avaliação quanto à presença ou ausência da sub-característica no software educacional. Existe um campo que identifica o responsável pelo preenchimento da sub-característica (E.U., Equipe de Usuários ou E.D., Equipe de Desenvolvimento) e os demais campos se referem ao Peso e a Nota (os quais o preenchimento será explicado na seção 3.1). Caso as informações descritas nos espaços reservados não sejam suficientes, o campo "Informações Adicionais" do mesmo formulário poderá ser utilizado. 
A característica Aspectos Educacionais, ausente na ISO 9126, foi incluída no processo para que o software seja avaliado enquanto produto de uma equipe de desenvolvimento e enquanto instrumento de ensino-aprendizagem e as suas subcaracterísticas representam esta ideia. Coerência está relacionada ao quão associado o software está a uma vertente pedagógica (construtivismo, interacionismo, por exemplo). A sub-característica Contextualização representa a aderência do software ao contexto em que o usuário está inserido, que pode ser entendido como contexto educacional. Motivação representa o quão é atrativo o software na atividade de expressar um conteúdo e avaliar o aluno em termos deste conhecimento. Satisfatoriedade vai indicar o nível de satisfação no uso do software, representada pela aderência do software aos objetivos pedagógicos definidos pelo professor e com que intensidade este software é utilizado na exposição de conteúdos e no estudo destes. Finalmente, com relação à Adequação à faixa etária tem-se a verificação da restrição de uso do software por um público com determinada idade, e se esta adequação também pode ser encarada com o objetivo de estimular o uso do software.

No que se refere aos papéis que participam da avaliação no Quali-EDU, além da Equipe de Desenvolvimento (E.D.) e da Equipe de Usuários (E.U.), existe o papel do Avaliador. A presença deste especialista em qualidade de software é crucial, visto que este será responsável por guiar o processo entre as partes envolvidas. Este é considerado o melhor entendedor do Quali-EDU, dando também o julgamento final, baseado no coletado pela E.U. e E.D.

\subsection{Fases do Processo de Avaliação}

O Quali-EDU composto por três fases, que foram definidas de acordo com a ISO/IEC 14598, conforme apresentado nas elipses da Figura 1.

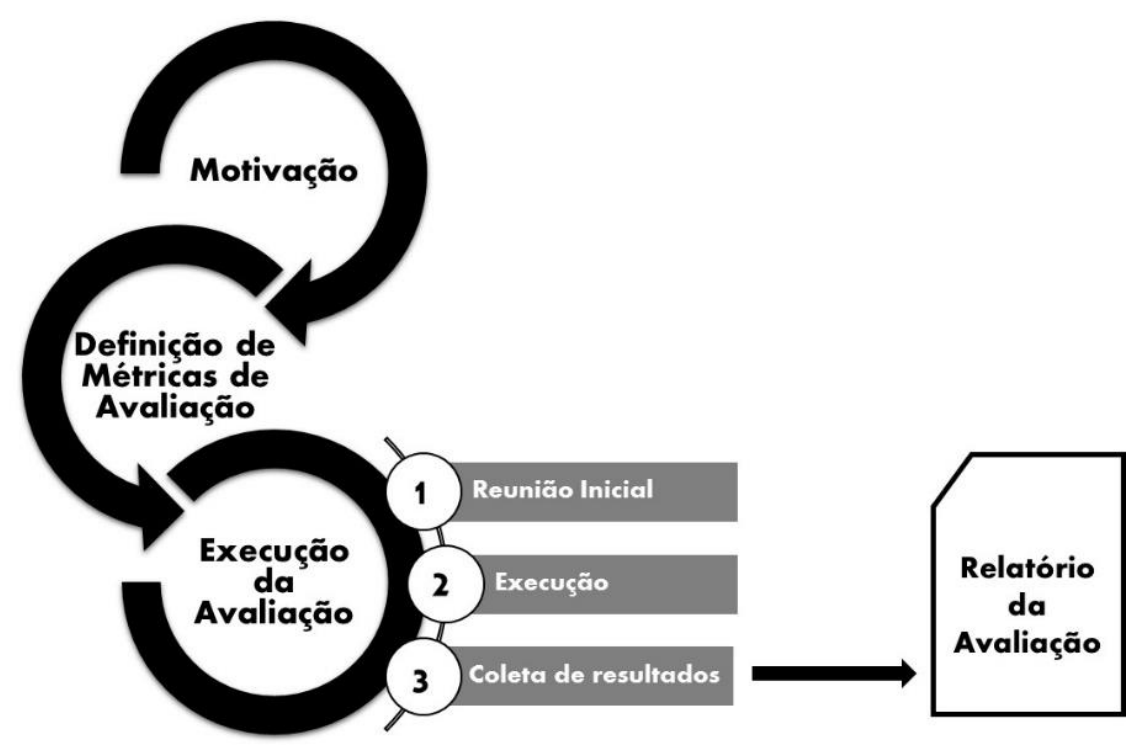

Figura 1. Fases do Quali-EDU

Na Fase 1 (Motivação), deve ser definido claramente qual o propósito da avaliação. Os propósitos de avaliação considerados pelo Quali-EDU estão em conformidade com a ISO/IEC 14598 (1998), que diz que o software pode ser avaliado 
enquanto fase de construção (protótipo), e também quando concluído. Na avaliação, podem ser destacados como propósitos a análise dos efeitos que o software irá despertar, verificação da necessidade de aprimoramentos e até substituição do software e obviamente, o julgamento sobre a aceitação do produto e quando este pode ser liberado. Ao fim desta fase, um documento será preenchido pelo avaliador geral, considerando o que foi coletado em reunião entre a E.U. e a E.D.

Uma vez definido o propósito da avaliação, na Fase 2 (Definição das métricas de avaliação), devem ser definidos valores e critérios de julgamento de cada uma das métricas. Segundo Cortes e Chiossi (2001), cada medida destas métricas contribui para um julgamento mais geral do produto, de acordo com o contexto do mesmo. Os procedimentos que sumarizam os resultados da avaliação em geral (cálculo da nota final), também são descritos nessa fase. O Quali-EDU apenas não contempla as métricas necessárias a um produto de software em construção (ponto destinado para trabalhos futuros).

A Fase 3 (Execução da Avaliação), é subdividida em 3 (três) momentos: Reunião Inicial, Execução e Coleta de Resultados, onde será aplicado o que foi especificado na fase anterior. No primeiro momento, o Avaliador apresenta à E.U. e à E.D. o Quali-EDU e os templates a serem preenchidos. A E.U. e a E.D. farão as escolhas dos pesos para cada sub-característica a ser avaliada, de acordo com o combinado entre estes. A reunião será concluída quando todos os pesos forem direcionados a cada questionamento dos templates.

No segundo momento (Execução), a E.U. e a E.D. munidas dos templates preenchidos com os pesos determinados anteriormente, terão acesso ao Software/Protótipo, e também, preferencialmente, ao documento de requisitos que apresenta o que é esperado do Software Educacional.

No terceiro momento, o avaliador executa a análise dos dados obtidos no momento anterior. Os templates possuem questionamentos que indicam o cumprime nto (ou não) de determinadas características a partir de suas sub-características. O peso direcionado a esse questionamento, pode ser 2,4 ou 6 , respectivamente, grau de importância baixo, médio e alto. Ea nota pode ser 0 (Não cumpriu com o questioname nto) ou 1 (Cumpriu com o questionamento). E, utilizando a média ponderada apresentada abaixo, obtém-se a nota final do software.

$$
\text { Nota Final }=\frac{S_{1} * P_{1}+S_{2} * P_{2}+S_{3} * P_{3}+\cdots+S_{n} * P_{n}}{\sum_{i=1}^{n} P i}
$$

De posse da Nota Final, será realizado o julgamento do software, conforme a Tabela 1.

Tabela 1. Classificação e julgamento do software [Marçal e Beuren 2009]

\begin{tabular}{|l|l|l|}
\hline Classificação & Valor da Nota Final & Julgamento \\
\hline Excelente & 0,91 a 1,0 & Aceito \\
\hline Bom & 0,76 a 0,90 & Aceito \\
\hline Satisfatório & 0,66 a 0,75 & Aceito com restrições \\
\hline Regular & 0,51 a 0,65 & Necessita verificações \\
\hline Insatisfatório & 0,0 à 0,5 & Rejeitado \\
\hline
\end{tabular}




\section{Avaliação utilizando o Quali -EDU}

Foram realizadas duas rodadas de avaliação que ocorreram na cidade de Sumé-PB, A primeira rodada contou com 5 (cinco) professores da rede municipal de ensino e a segunda rodada com 13 (treze) alunos do Campus de Sumé - Paraiba.

Com relação à primeira rodada de avaliações, dos professores convidados, dois lecionam matemática no ensino fundamental II e possuem graduação em Licenciatura Plena em Matemática. Os outros três possuem graduação em Licenciatura Plena em Pedagogia. A segunda rodada de avaliações foi realizada por 13 (treze) alunos, acadêmicos do curso de Licenciatura em Educação do Campo, do Centro de Desenvolvimento Sustentável do Semiárido - Campus de Sumé.

O software a ser avaliado nas duas rodadas foi o jogo Tux of Math Command ${ }^{1}$ (Tux Math), que foi desenvolvido pela empresa Tux4Kids, utilizando a linguagem $\mathrm{C} \mathrm{e}$ para os padrões gráficos o SDL (Simple DirectMedia Layer). Destinado a computadores, inicialmente foi produzido para sistema operacional Linux, porém atualmente, disponíve 1 para outros sistemas operacionais.

O principal objetivo do TuxMath é que o jogador proteja os seus iglus de possíveis cometas que sempre aparecem na tela, caindo em direção aos mesmos. Para que o cometa seja destruído, o usuário necessitará solucionar as questões matemáticas que aparecem na tela, digitando a solução e pressionando a tecla "enter". Após essa ação do usuário, caso a solução esteja correta, o cometa será destruído, caso contrário, o disparo do laser não atingirá nenhum dos cometas que estão caindo, denotando o erro por parte do aluno.

O TuxMath foi escolhido como software educacional para a avaliação inicial do Quali-EDU porque é um software educacional que faz parte do Linux Educacional, que é o sistema operacional instalado nas máquinas presentes nos laboratórios das escolas públicas brasileiras. Além disso, o TuxMath é um software fácil de utilizar o que simplifica o treinamento da equipe no uso do software, agilizando a avaliação.

\subsection{Execução, Coleta e Análise dos Resultados}

\subsubsection{Primeira Rodada}

Após a realização da reunião inicial, descrita na fase de execução do Quali-EDU, os professores definiram o propósito da avaliação. Considerando que o software já estava pronto, o propósito definido foi "Decidir sobre a necessidade aprimoramentos/substituição do software".

Os 5 (cinco) professores convidados, utilizando os templates do Quali-EDU, iniciaram a discussão (sem a presença do avaliador) sobre os pesos que seriam destinados às questões sob a responsabilidade da E.U, atribuíram os pesos para cada característica e sub-característica escolhida e realizaram a sua avaliação individual do software. Para a característica Usabilidade foram escolhidas as sub-características Inteligibilidade, Apreensibilidade, Operacionalidade e Atratividade. Para a característica Funcionalida de foram escolhidas as sub-características Adequação e Acurácia. Com relação à

\footnotetext{
${ }^{1}$ http://pt.wikipedia.org/wiki/TuxMath
} 
Portabilidade as sub-características selecionadas para a avaliação do software foram Adaptabilidade, Capacidade de ser instalado, Coexistência e Capacidade para substituir. Finalmente, para a característica Aspectos educacionais foram utilizadas todas as subcaracterísticas (Coerência, Contextualização, Motivação, Satisfatoriedade e Adequação à faixa etária).

Após a fase da execução da avaliação, iniciou-se a fase da coleta de dados, que é sumarizada na Tabela 2.

Tabela 2. Pesos e notas provenientes da avaliação dos professores da E.U.

\begin{tabular}{|l|c|ccccc|}
\cline { 3 - 7 } \multicolumn{2}{c|}{} & \multicolumn{5}{c|}{ Usuários } \\
\hline Sub-características & \multirow{2}{*}{ Pesos } & U1 & U2 & U3 & U4 & U5 \\
\hline Inteligibilidade & 5 & 1 & 0 & 0 & 1 & 0 \\
Apreensibilidade & 3 & 0 & 1 & 1 & 1 & 0 \\
Operacionalidade & 5 & 1 & 0 & 0 & 0 & 1 \\
Atratividade & 3 & 0 & 0 & 1 & 1 & 1 \\
\hline Adequação & 5 & 0 & 1 & 0 & 1 & 1 \\
Acurácia & 5 & 0 & 1 & 0 & 0 & 1 \\
\hline Capacidade de ser instalado & 3 & 1 & 0 & 1 & 0 & 0 \\
Capacidade para substituir & 1 & 0 & 0 & 1 & 1 & 1 \\
\hline Coerência & 5 & 0 & 1 & 1 & 0 & 0 \\
Contextualização & 5 & 1 & 0 & 0 & 1 & 1 \\
Motivação & 5 & 0 & 1 & 0 & 1 & 0 \\
Satisfatoriedade & 5 & 1 & 0 & 1 & 1 & 0 \\
Adequação à faixa etária & 5 & 1 & 0 & 0 & 1 & 1 \\
\hline \multicolumn{1}{|c|}{ TOTAIS } & $\mathbf{5 5}$ & $\mathbf{0 , 5 1}$ & $\mathbf{0 , 3 4}$ & $\mathbf{0 , 2 9}$ & $\mathbf{0 , 5 4}$ & $\mathbf{0 , 4 3}$ \\
\hline
\end{tabular}

O último momento da fase de execução da avaliação foi a coleta dos templates pelo avaliador, com as seguintes notas: 0,$51 ; 0,34 ; 0,29 ; 0,54 ; 0,43$, atribuídas por cada um dos usuários, respectivamente.

Conforme explicitado na seção 3.2, após o cálculo da média ponderada, foi obtido o valor 0,56. Assim, de acordo com a Tabela 1, o TuxMath recebeu a classificação de "Regular", ou seja, necessitaria de verificações antes de chegar ao Mercado/Salas de aula.

\subsubsection{Segunda Rodada}

Da mesma maneira que ocorreu na primeira rodada de avaliações, houve uma reunião inicial sem a presença do Avaliador, para que fosse definido o propósito da avaliação. Com a reunião inicial finalizada, os alunos definiram o propósito da avaliação, que foi "Decidir sobre a necessidade aprimoramentos/substituição do software, de acordo com o contexto em que é utilizado".

Assim como os professores, os alunos convidados preencheram os templates do Quali-EDU, com os pesos para cada sub-característica sendo atribuídos por cada um deles. Os alunos também avaliaram o software TuxMath.

No momento da Execução, as notas foram atribuídas. Os alunos avaliaram o mesmo conjunto de características e sub-características que os professores. A Tabela 3 apresenta a avaliação de cada aluno para cada um dos itens escolhidos para a avaliação do software. 
Tabela 3. Pesos e notas provenientes da avaliação dos professores da E.U.

\begin{tabular}{|c|c|c|c|c|c|c|c|c|c|c|c|c|c|c|}
\hline \multirow[b]{2}{*}{ Sub-características } & \multirow[b]{2}{*}{ Pesos } & \multicolumn{13}{|c|}{ Usuários } \\
\hline & & U1 & $\mathbf{U} \mathbf{2}$ & $\mathbf{U 3}$ & U4 & U5 & U6 & U7 & U8 & U9 & U10 & U11 & U12 & U13 \\
\hline Inteligibilidade & 6 & 0 & 0 & 0 & 0 & 1 & 0 & 0 & 1 & 1 & 1 & 1 & 1 & 1 \\
\hline Apreensibilidade & 6 & 0 & 0 & 0 & 0 & 1 & 0 & 1 & 1 & 1 & 1 & 1 & 1 & 0 \\
\hline Operacionalidade & 4 & 1 & 0 & 1 & 0 & 1 & 0 & 1 & 1 & 1 & 1 & 1 & 1 & 1 \\
\hline Atratividade & 6 & 0 & 0 & 1 & 0 & 1 & 0 & 1 & 1 & 1 & 1 & 1 & 1 & 1 \\
\hline Adequação & 4 & 1 & 1 & 1 & 1 & 1 & 0 & 0 & 1 & 1 & 1 & 1 & 1 & 1 \\
\hline Acurácia & 6 & 1 & 1 & 1 & 1 & 1 & 1 & 0 & 1 & 0 & 1 & 1 & 1 & 1 \\
\hline Adaptabilidade & 6 & 0 & 0 & 1 & 1 & 0 & 0 & 0 & 1 & 1 & 1 & 1 & 1 & 0 \\
\hline $\begin{array}{l}\text { Capacidade de ser } \\
\text { instalado }\end{array}$ & 6 & 0 & 1 & 1 & 1 & 1 & 1 & 0 & 1 & 0 & 1 & 1 & 1 & 0 \\
\hline Coexistência & 2 & 0 & 0 & 0 & 0 & 0 & 1 & 0 & 1 & 0 & 0 & 0 & 0 & 0 \\
\hline $\begin{array}{l}\text { Capacidade para } \\
\text { substituir }\end{array}$ & 4 & 0 & 0 & 0 & 0 & 0 & 1 & 0 & 1 & 0 & 1 & 1 & 0 & 1 \\
\hline Coerência & 6 & 1 & 0 & 0 & 0 & 1 & 0 & 0 & 1 & 1 & 1 & 1 & 0 & 1 \\
\hline Contextualização & 6 & 0 & 0 & 0 & 0 & 1 & 0 & 0 & 1 & 1 & 1 & 1 & 1 & 0 \\
\hline Motivação & 6 & 0 & 0 & 0 & 0 & 1 & 0 & 1 & 1 & 1 & 1 & 1 & 0 & 1 \\
\hline Satisfatoriedade & 4 & 0 & 0 & 0 & 0 & 1 & 0 & 0 & 1 & 0 & 1 & 0 & 0 & 1 \\
\hline $\begin{array}{l}\text { Adequação à faixa } \\
\text { etária }\end{array}$ & 6 & 1 & 0 & 0 & 1 & 0 & 0 & 0 & 1 & 1 & 1 & 1 & 1 & 1 \\
\hline TOTAIS & 78 & $\mathbf{0 , 3 3}$ & 0,21 & 0,41 & 0,36 & $\mathbf{0 , 7 7}$ & 0,23 & 0,28 & 1 & 0,72 & 0,72 & 0,92 & $\mathbf{0 , 7 2}$ & 0,67 \\
\hline
\end{tabular}

Calculada a média ponderada, foi obtido o valor 0,27. Este valor, de acordo com a Tabela 1, recebeu a classificação de "Insatisfatório", o que significa que do ponto de vista dos alunos, é um software considerado Rejeitado para uso em sala de aula.

\section{Considerações Finais e Trabalhos Futuros}

Existem disponíveis diversos modelos de avaliação de qualidade de software, reconhecidos internacionalmente, por entidades oficiais. No entanto, a pesquisa realizada reconheceu poucos que possuem nas suas etapas a inclusão de prerrogativas que permitam a avaliação da qualidade do software educacional, considerando suas especificidades.

Portanto, ao adicionar às características existentes na norma ISO/IEC 9126 a característica "Aspectos Educacionais", e levar o processo à aplicação, foi possível observar que se houvesse a avaliação do produto de software educacional antes de utilizalo, considerando o contexto do usuário que irá utiliza-lo, seria possível um melhor aproveitamento deste por parte dos alunos.

Considerando o aspecto relacionado à avaliação do produto de software educacional em desenvolvimento, também presente no Quali-EDU, os softwares educacionais, após a avaliação preliminar proporcionada pelo mencionado processo, poderiam ter seu desenvolvimento direcionado às características inerentes aos Aspectos Educacionais do contexto a ser aplicado. Isto pode vir a permitir que o software educacional realmente exerça seu papel educacional, de forma eficiente e natural, propiciando ao aluno uma nova possibilidade de aprender e de adquirir conhecimento, atingindo, portanto, a finalidade para a qual foi proposto. Esta é uma necessidade que se verificou na avaliação do TuxMath, quando aplicado o Quali-EDU em condições reais, uma vez que foi julgado como "Regular" pelos professores e "Insatisfatório" pelos alunos, o que, pode indicar a necessidade de ajustes para que possa ser utilizado. 
Como trabalhos futuros, sugere-se a criação de métricas para complementar o que foi proposto na seção 3.1 e pretende-se incluir nas pesquisas a norma ISO/IEC 25000:2014, mais conhecida como SQuaRE, sucessora da ISO/IEC 9126, que trata diretamente dos requisitos de qualidade e avaliação de softwares.

\section{Referências}

Cortes, M e Chiossi, T. (2001) "Modelos de Qualidade de Software". Campinas, SP, Editora da UNICAMP, IC.

Coutinho, C. e Lisbôa, E. (2011) "Sociedade da informação, do conhecimento e da aprendizagem: desafios para educação no século XXI" In: Revista de Educação, Vol XVIII, no 1, p. 5-22.

Fang, H. (2008) "Modeling and Analysis for Educational Software Quality Hierarchy Triangle" In: Proceedings of Seventh International Conference on Web-based Learning, Zhejiang, China.

Giraffa, L. (2000) "A Comunidade de Informática Educativa Brasileira: Perspectiva Histórica e Pesquisa", Painel Integrado de Palestras e Debates. In: VI Workshop de Informática na Escola, Curitiba, Brasil.

ISO/IEC 9126 (2002) “Software Engineering - Product Quality”.

ISO/IEC 14598 (1998) "Information Technology - Evaluation of Software Products".

Kelly, D. (2008) "Innovative Standards for Innovative Software" Computer. July, pp. 8889.

Litchfield, A. (1994) "Interface communication management: A user centred multimedia design model" In: C. McBeath and R. Atkinson (Eds), Proceedings of the Second International Interactive Multimedia Symposium, 298-303. Perth, Western Australia, 23-28 January. Promaco Conventions.

Lou Y., Abrami P.C. e D'apollonia S. (2001) "Small group and individual learning with technology: a meta-analysis", In: Review of Educacional Research. vol. 71 no. 3 449521.

Marçal, E. e Beuren, I.M. (2009) "Auditoria da qualidade de um software de contabilidade", Gestão \& $\quad$ Regionalidade, v. 23. http//seer.uscs.edu.br/index.php/revista_gestao/article/download/68/31, Junho.

Nori, K., Reddy, R. e Chimalakonda, S. (2014) "Challenges for Software Engineering in Educational Technologies", In: International Conference on Contemporary Computing and Informatics (IC3I), Karnataka, India.

Nussbaum, M. e Infante, C. (2013) "Guidelines for Educational Software Design that Consider the Interests and Needs of Teachers and Students", In: 13th International Conference on Advanced Learning Technologies, Beijing, China.

Rocha, A. R. e Campos, G. H. B. (1993) "Avaliação da Qualidade de Software Educacional", Em Aberto, Brasilia, ano 12, n.57, jan./mar.

Scardamalia, M. e Bereiter C. (1996) "Engaging Students in a Knowledge Society" Educational Leadership, 54(3), 6-10. 